\title{
Pseudotrienic Acid A and B, two Bioactive Metabolites from Pseudomonas sp. MF381-IODS
}

Anton Pohanka, ${ }^{* \dagger}$ Anders Broberg, ${ }^{\dagger}$ Maria Johansson, ${ }^{*}$ Lennart Kenne, ${ }^{\dagger}$ and Jolanta Levenfors ${ }^{s, \perp}$

Department of Chemistry, Swedish University of Agricultural Sciences, P.O. Box 7015, SE-750

07 Uppsala, Sweden, Department of Plant Pathology and Biological Control Unit, Swedish

University of Agricultural Sciences, P.O. Box 7035, SE-750 07 Uppsala, Sweden, and Agrivir AB, P.O. Box 914, SE-751 09 Uppsala, Sweden

NMR and MS data for DDR methanol adduct and pyrrolnitrine

Page S2

NMR spectra for 2:

DQF-COSY

Page S3

TOCSY

Page S4

NOESY

Page S5

DEPT-HSQC

Page S6

HMBC

Page S7

Experimental data for lactone formation of $\mathbf{2}$ to $\mathbf{2 a}$

Page S8

${ }^{1} \mathrm{H}$ NMR spectra of lactone formation analysis

Page S9

Co-authors are listed in alphabetical order. "To whom correspondence should be addressed. Tel: +46-18-671549. Fax: +46-18-673476. E-mail: Anton.Pohanka@kemi.slu.se.

'Department of Chemistry, Swedish University of Agricultural Sciences

*Plant Pathology and Biological Control Unit, Swedish University of Agricultural Sciences

${ }^{\S}$ Agrivir $\mathrm{AB}$

${ }^{\perp}$ Present address: The MASE Laboratories, P.O. Box 148, SE-751 04 Uppsala, Sweden 
DDR methanol adduct (3a): White powder; ${ }^{1} \mathrm{H} \mathrm{NMR}\left(\mathrm{CDCl}_{3}, 600 \mathrm{MHz}\right) \delta 7.53(1 \mathrm{H}, \mathrm{s}, \mathrm{H}-25)$, $6.77(1 \mathrm{H}, \mathrm{ddd}, J=15.6,6.5,6.5 \mathrm{~Hz}, \mathrm{H}-3), 6.60(1 \mathrm{H}, \mathrm{dd}, J=15.0 \mathrm{~Hz}, 10.8, \mathrm{H}-20), 6.38(1 \mathrm{H}, \mathrm{d}, J$ $=15.0 \mathrm{~Hz}, \mathrm{H}-21), 6.26(1 \mathrm{H}, \mathrm{s}, \mathrm{H}-23), 6.10(1 \mathrm{H}, \mathrm{d}, J=10.8 \mathrm{~Hz}, \mathrm{H}-19), 5.70(1 \mathrm{H}, \mathrm{d}, J=15.6 \mathrm{~Hz}$, H-2), $5.51(1 \mathrm{H}, \mathrm{dd}, J=15.6,9.0 \mathrm{~Hz}, \mathrm{H}-9), 5.15(1 \mathrm{H}, \mathrm{dd}, J=15.6,8.4 \mathrm{~Hz}, \mathrm{H}-10), 4.80$ (1H, dd, $J$ = 10.2, $3 \mathrm{~Hz}, \mathrm{H}-15), 3.71(3 \mathrm{H}, \mathrm{s}, \mathrm{H}-36), 3.25(1 \mathrm{H}, \mathrm{d}, J=10.8 \mathrm{~Hz}, \mathrm{H}-17), 3.18(1 \mathrm{H}, \mathrm{m}, \mathrm{H}-7), 3.17$ (3H, s, H-32), $3.14(1 \mathrm{H}, \mathrm{d}, J=8.4 \mathrm{~Hz}, \mathrm{H}-11), 3.04(1 \mathrm{H}, \mathrm{dd}, J=9.6, \sim 1,5 \mathrm{~Hz}, \mathrm{H}-13), 2.54(1 \mathrm{H}, \mathrm{dd}$, $J=15.6,7.2 \mathrm{~Hz}, \mathrm{H}-27 \mathrm{a}), 2.46$ (3H, s, H-35), 2.44 (1H, m, H-4a), $2.40(1 \mathrm{H}, \mathrm{dd}, J=15.6,7.2 \mathrm{~Hz}$, H-27b), 2.23 (1H, bm, H-5), 2.15 (3H, s, H-34), 2.10 (1H, m, H-16), 2.08 (1H, m, H-4b), 2.05 (1H, m, H-8), 1.98 (1H, m, H-14a), 1.86 (3H, s, H-33), 1.80 (1H, m, H-14b), 1.78 (1H, M, H-6a), 1.35 (3H, s, H-30), 1.07 (1H, m, H-6b), 1.05 (3H, d, $J=6.6 \mathrm{~Hz}, \mathrm{H}-29), 1.00$ (3H, d, $J=6.6 \mathrm{~Hz}$, $\mathrm{H}-31) ;{ }^{13} \mathrm{C} \mathrm{NMR}\left(\mathrm{CDCl}_{3}, 150 \mathrm{MHz}\right) \delta 173.9$ (C, C-28), 165.5 (C, C-1), 161.4 (C, C-26), 146.5 (CH, C-3), 141.3 (CH, C-9), 138.4 (C, C-24), 137.6 (CH, C-21), 137.0 (C, C-22), 136.7 (C, C18), 135 (CH, C-25), 129.7 (CH, C-19), 125.6 (CH, C-10), 124.9 (CH, C-2), 124.3 (CH, C-20), 120.7 (CH, C-23), 89.4 (CH, C-17), 78.7 (CH, C-13), 74.4 (CH, C-7), 73.5 (CH, C-15), 66.0 (C, C-12), 63.9 (CH, C-11), $56.8\left(\mathrm{CH}_{3}, \mathrm{C}-32\right), 51.8\left(\mathrm{CH}_{3}, \mathrm{C}-36\right), 45.7(\mathrm{CH}, \mathrm{C}-8), 40.8\left(\mathrm{CH}_{2}, \mathrm{C}-27\right)$, $39.5(\mathrm{CH}, \mathrm{C}-16), 38.0\left(\mathrm{CH}_{2}, \mathrm{C}-6\right), 37.7\left(\mathrm{CH}_{2}, \mathrm{C}-4\right), 32.2(\mathrm{CH}, \mathrm{C}-5), 32.0\left(\mathrm{CH}_{2}, \mathrm{C}-14\right), 17.3\left(\mathrm{CH}_{3}\right.$, C-29), $14.6\left(\mathrm{CH}_{3}, \mathrm{C}-34\right), 13.9\left(\mathrm{CH}_{3}, \mathrm{C}-35\right), 11.7\left(\mathrm{CH}_{3}, \mathrm{C}-33\right), 11.4\left(\mathrm{CH}_{3}, \mathrm{C}-30\right), 10.4\left(\mathrm{CH}_{3}, \mathrm{C}-\right.$ 31); ESIMS $m / z 664.3[\mathrm{M}+\mathrm{Na}]^{+}$.

Pyrrolnitrine (4): White powder; ${ }^{1} \mathrm{H}$ NMR $\left(\mathrm{CDCl}_{3}, 600 \mathrm{MHz}\right) \delta 8.29(1 \mathrm{H}, \mathrm{br} \mathrm{s}, \mathrm{NH}), 7.53(1 \mathrm{H}$, m, H-4'), 7.45 (1H, m, H-5), 7.43 (1H, m, H-6'), 6.85 (1H, m, H-2), 6.84 (1H, m, H-5); ${ }^{13} \mathrm{C}$ NMR $\left(\mathrm{CDCl}_{3}, 150 \mathrm{MHz}\right) \delta 149.6\left(\mathrm{C}, \mathrm{C}-2^{\prime}\right), 130.6\left(\mathrm{CH}, \mathrm{C}-4\right.$ '), $130.2\left(\mathrm{CH}, \mathrm{C}-5^{\prime}\right), 129.0\left(\mathrm{CH}, \mathrm{C}-6^{\prime}\right)$, 128.2 (C, C-1'), 125.3 (C, C-3'), 117.5 (CH, C-5), 116.7 (CH, C-2), 115.7 (C, C-4), 112.5 (C, C3); ESIMS $m / z 279[\mathrm{M}+\mathrm{Na}]^{+}$. 
DQFCOSY spectrum of $\mathbf{1}\left(\mathrm{CD}_{3} \mathrm{OD}, 600 \mathrm{MHz}\right)$<smiles>CCCCCCCCC[C@H](O)/C=C/C(C)=C/CC(=O)NC[C@H](O)[C@H](C)C(=O)NCC/C=C/C=C/C=C/C(=O)O</smiles>

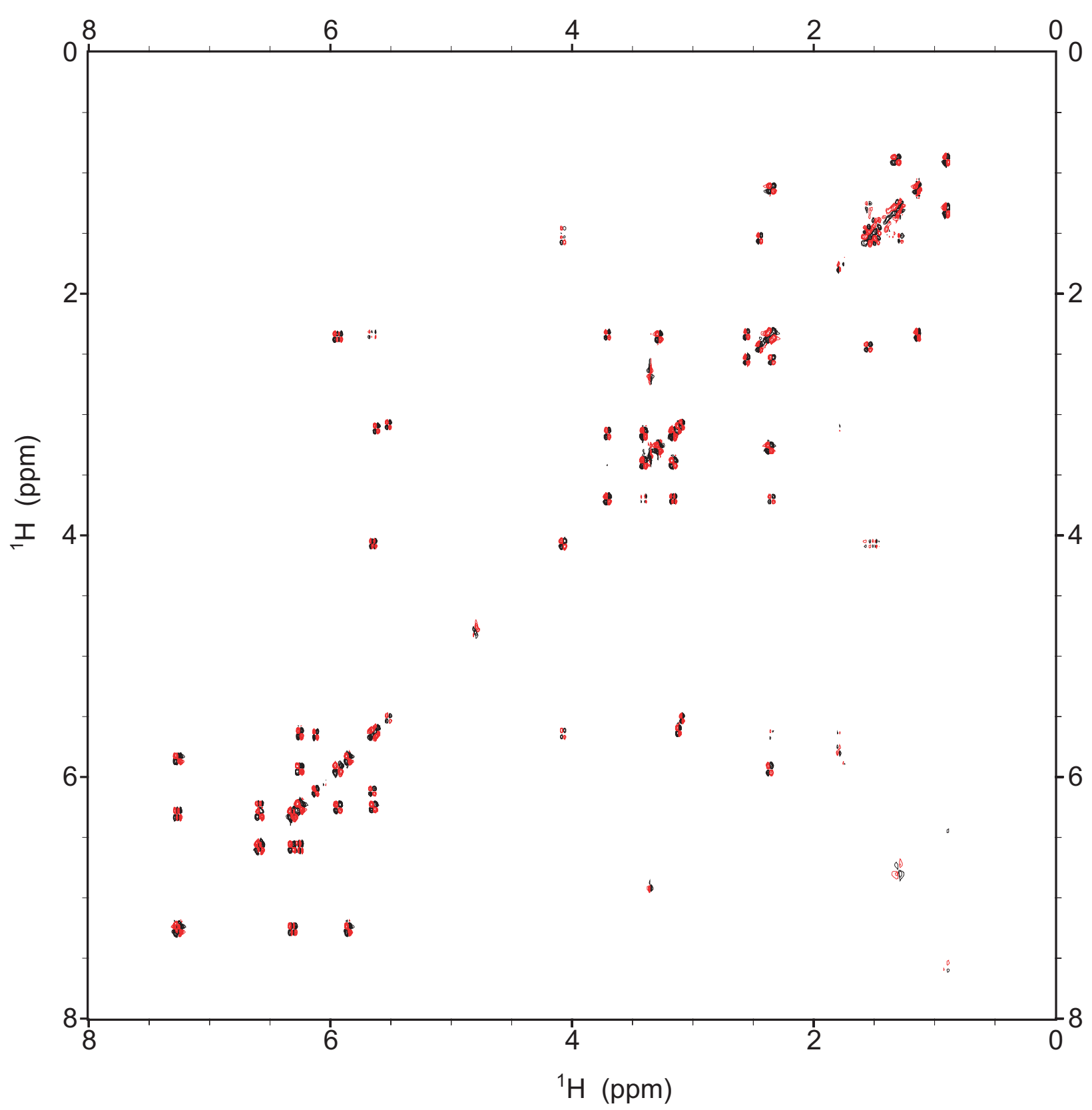


TOCSY spectrum of $\mathbf{1}\left(\mathrm{CD}_{3} \mathrm{OD}, 600 \mathrm{MHz}\right)$<smiles>CCCCCCCCC[C@H](O)/C=C/C(C)=C/CC(=O)NC[C@@H](O)[C@H](C)C(=O)NCC/C=C/C=C/C=C/C(=O)O</smiles>

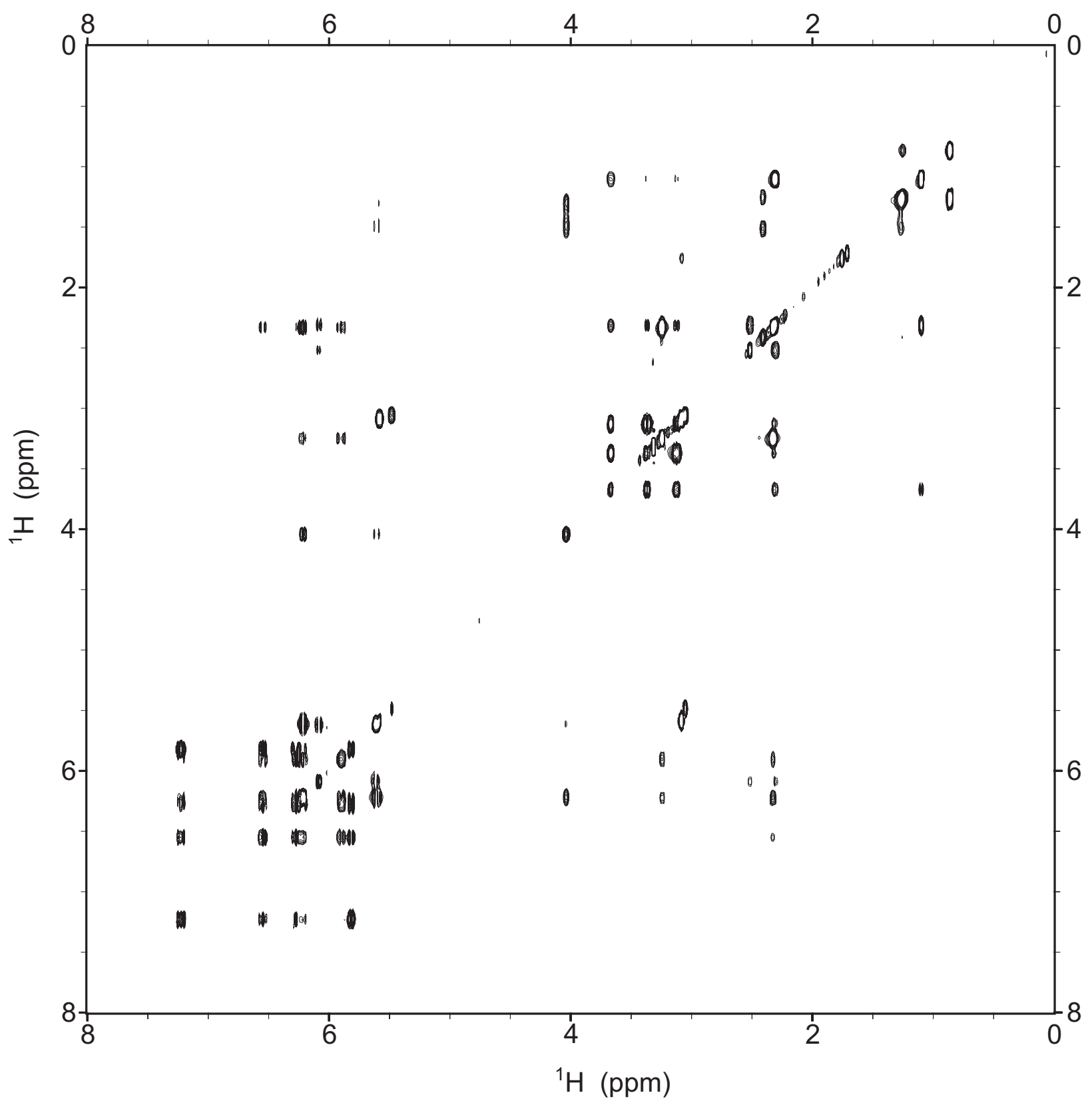


NOESY spectrum of $\mathbf{1}\left(\mathrm{CD}_{3} \mathrm{OD}, 600 \mathrm{MHz}\right)$

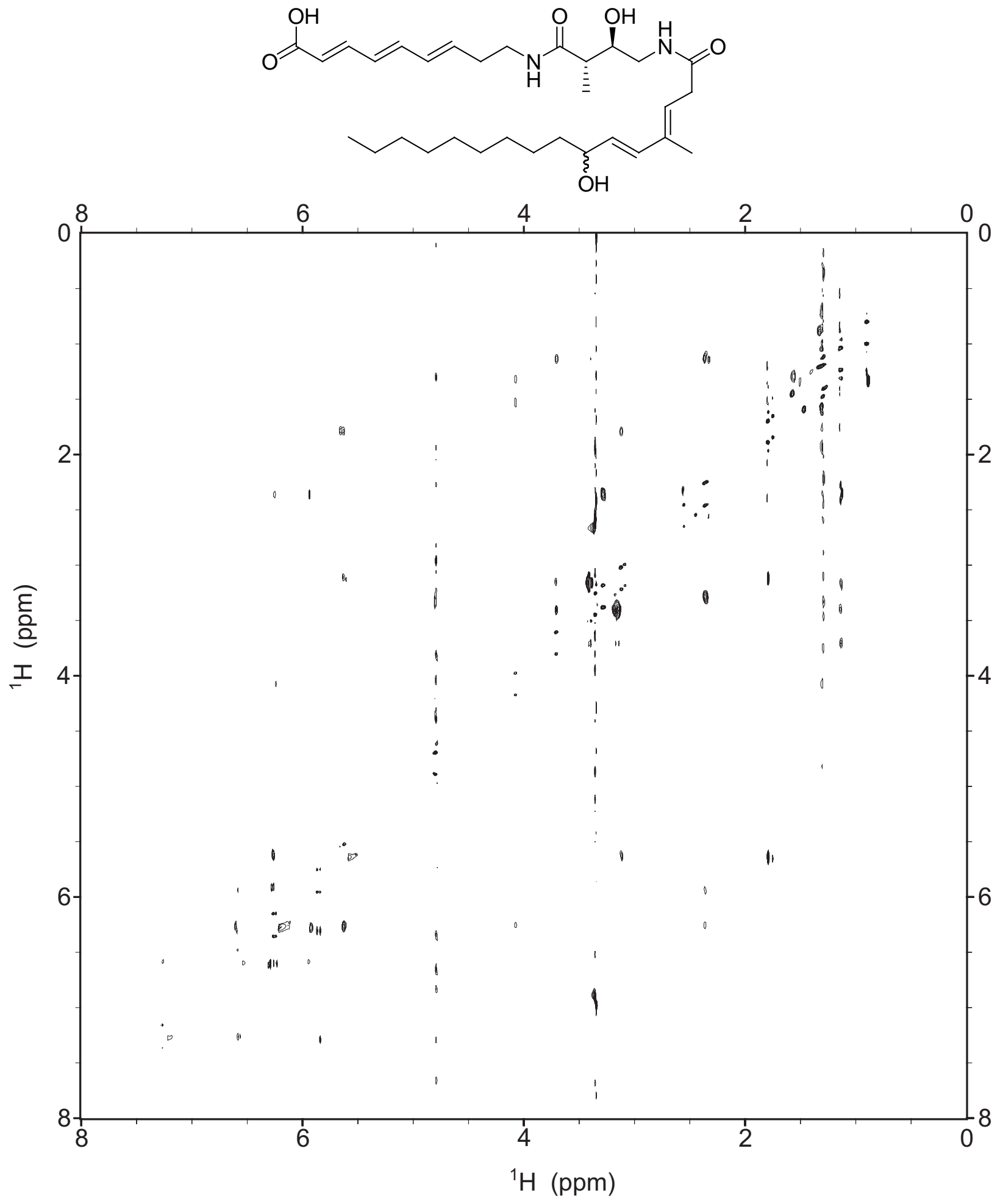


DEPTHSQC spectrum of $\mathbf{1}\left(\mathrm{CD}_{3} \mathrm{OD}, 150 / 600 \mathrm{MHz}\right)$

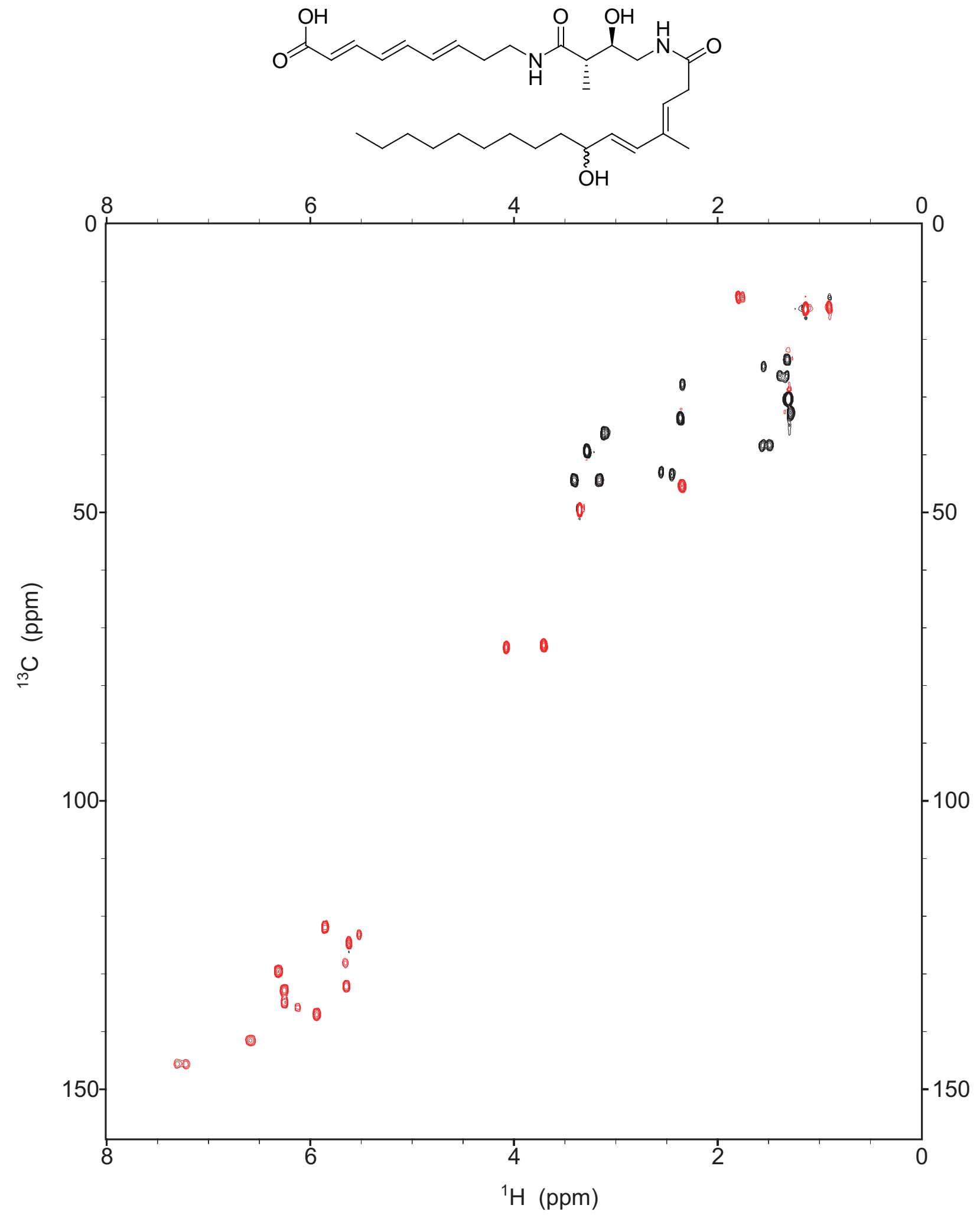


HMBC spectrum of $1\left(\mathrm{CD}_{3} \mathrm{OD}, 150 / 600 \mathrm{MHz}\right)$

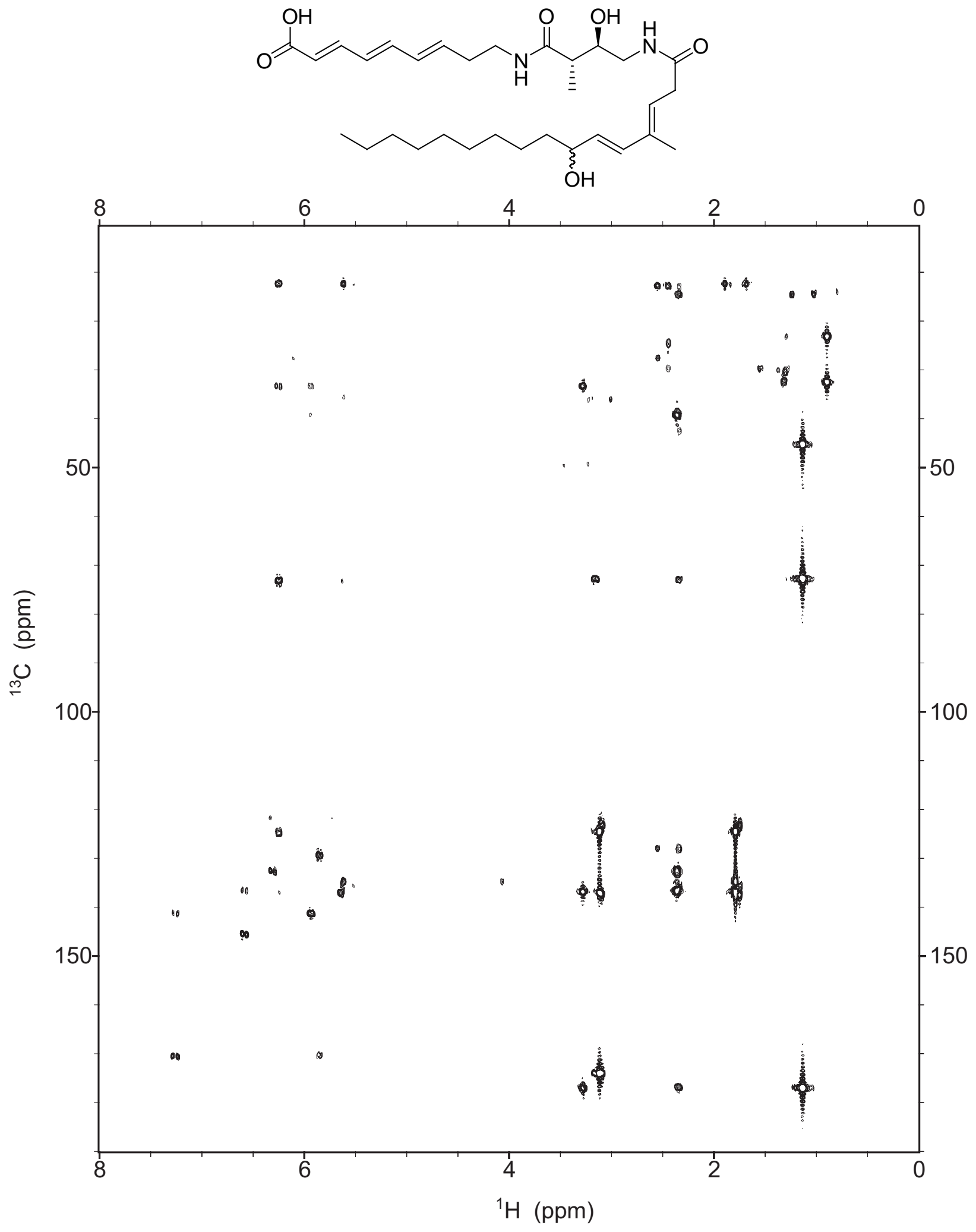




\section{Lactone formation of 2 to $2 \mathrm{a}$ :}

About $1 \mathrm{mg}$ of 2 was dissolved in $950 \mu \mathrm{L} 1 \%$ TFA in $50 / 50 \mathrm{MeCN} / \mathrm{H}_{2} \mathrm{O}$ and analyzed at 30 min intervals by RP-HPLC with UV-detection at $310 \mathrm{~nm}(41 \%$ A for $15 \mathrm{~min}, 41 \%$ to $100 \%$ A in $5 \mathrm{~min}, 100 \%$ A for $10 \mathrm{~min}$; A: aqueous $10 \mathrm{mM}$ ammonium acetate at $\mathrm{pH}$ 4.5, $\mathrm{B}: \mathrm{CH}_{3} \mathrm{CN}$ ). Compound 2 eluted at $t_{\mathrm{R}}=14.1 \mathrm{~min}$ and a peak at $t_{\mathrm{R}}=22.7 \mathrm{~min}$ appeared immediately at $\mathrm{t}=0$ and reached maximum area after $60 \mathrm{~min}$. This also correlated with disappearance of the peak at $t_{\mathrm{R}}=14.1 \mathrm{~min}$. When subjected to LC-ESIMS in negative mode, the peak at $t_{\mathrm{R}}=22.7 \mathrm{~min}$ correlated to $\mathrm{m} / z$ of 499.5 [M-H] , i.e. corresponding to the lactone 2a. The peak of 2a was isolated and subjected to ${ }^{1} \mathrm{H} N M R$ and $\mathrm{COSY}$ in $\mathrm{CD}_{3} \mathrm{CN} / \mathrm{D}_{2} \mathrm{O} 50 / 50$ (see page $\mathrm{S}$, spectrum C). To verify the lactone formation, ${ }^{1} \mathrm{H}$ NMR and COSY was run on $0.5 \mathrm{mg}$ of 2 dissolved in $500 \mu \mathrm{L} \mathrm{CD}_{3} \mathrm{CN} / \mathrm{D}_{2} \mathrm{O}$ $50 / 50$ (see page S9, spectrum A). Then TFA was added to a concentration of 5\% and the mixture was monitored by ${ }^{1} \mathrm{H}$ NMR and COSY. After five min about $60 \%$ of the material had formed lactones (see page S9, spectrum B). Although the high TFA content influenced the HDO resonance the chemical shifts and connectivities could be verified by comparison with the isolated lactone sample from the HPLC experiment and the control spectra acquired prior to addition of TFA. 
${ }^{1} \mathrm{H}$ NMR spectra (600 MHz) of lactone formation analysis with the H-20 (2) and H-16 (2a) resonance marked. A: ${ }^{1} \mathrm{H}$ NMR of $2\left(\mathrm{CD}_{3} \mathrm{CN} / \mathrm{D}_{2} \mathrm{O} 50 / 50\right)$; B: ${ }^{1} \mathrm{H}$ NMR of 2 five min after addition of $5 \%$ TFA $\left(\mathrm{CD}_{3} \mathrm{CN} / \mathrm{D}_{2} \mathrm{O} 50 / 50,5 \% \mathrm{TFA}\right) ; \mathrm{C}:{ }^{1} \mathrm{H}$ NMR of $\mathbf{2 a}\left(\mathrm{CD}_{3} \mathrm{CN} / \mathrm{D}_{2} \mathrm{O} 50 / 50\right)$

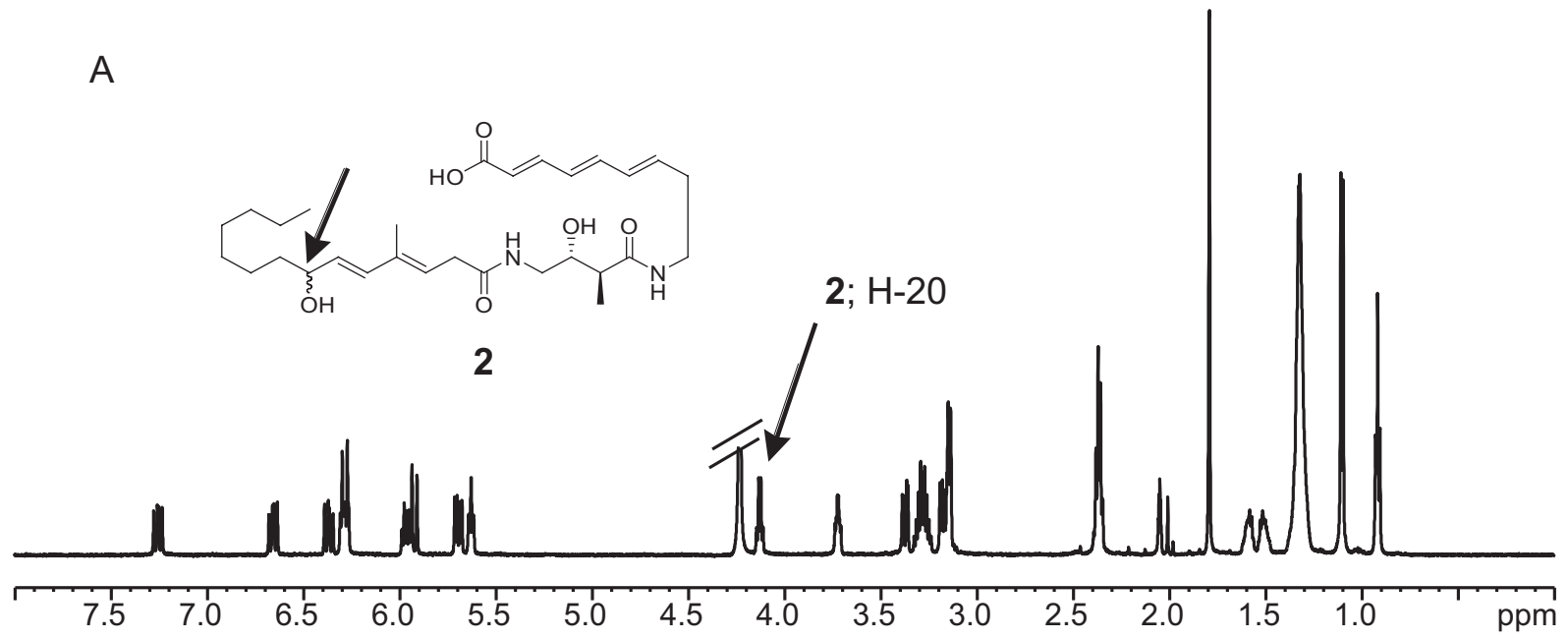

B
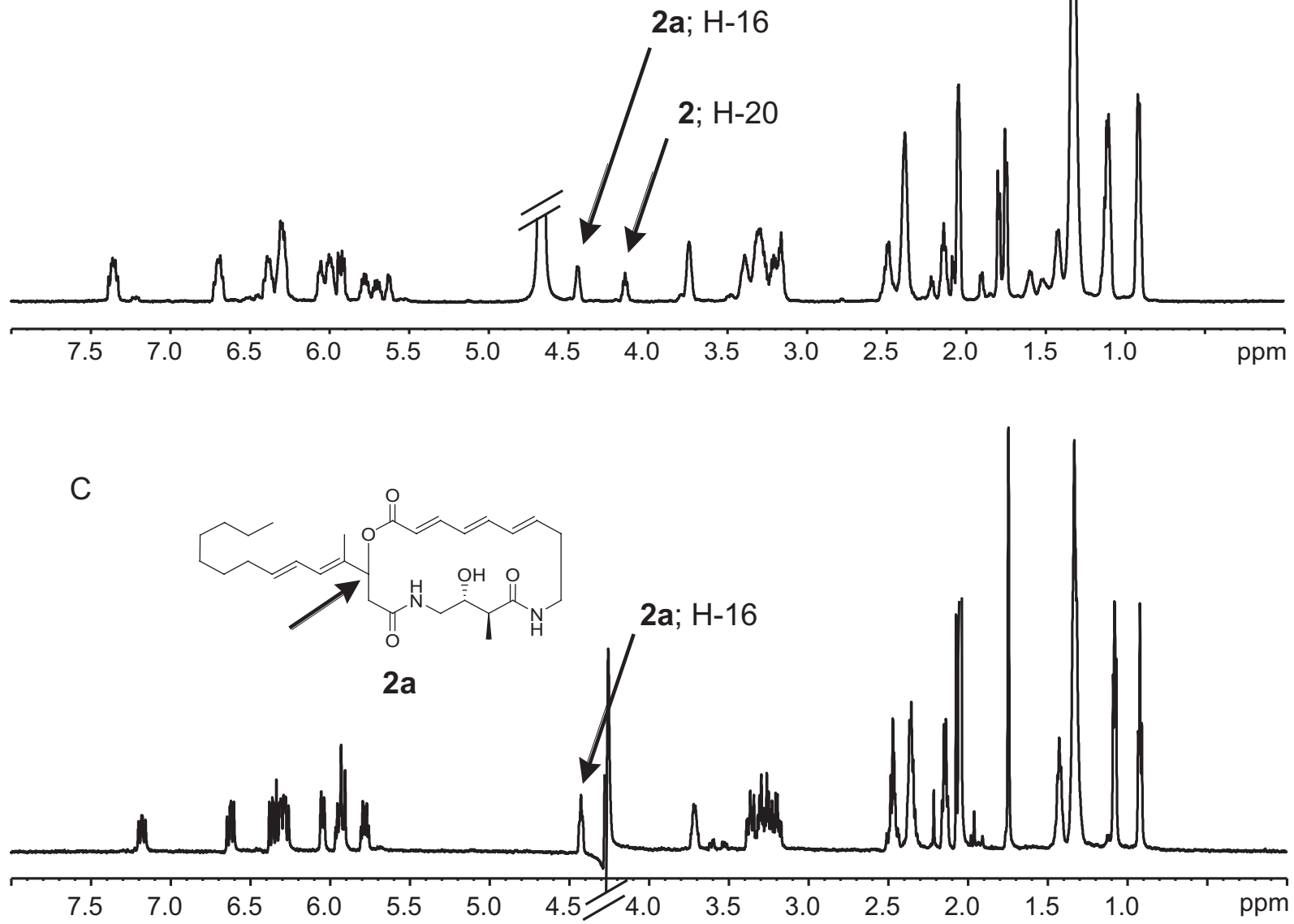